[4] G. Halisz and P. Turán, On the distribntion of roots of Riemann zeta and allied functions I-II, J. Number Theory 1 (1969), pp. 121-137; Acta Math. Hung. 21 (1970), pp. 403-419.

[5] M. Huxley, On the aifference between eonsecutive primes, Inventionos Math. 15 (1972), pp. 164-170.

[6-8] - Large values of Dirichlet polynomials, Aeta Arith. 24 (1973), pp. 329-346; II, ibid., 27 (1975), pp. 159-169; III, ibid., 26 (1975), pp. 435-44.4.

[9] - An imperfect hybricl zero-ilensity theorem, J. Tondon Math. Soc. (2) 13 (1.976), pp. $53-56$.

[10] M. Jutila, On a density theorem of H.L. Montgomery for $L$-janctions, Ann. Acad. Sci. Fonn. sor. A I 520 (1972). $13 \mathrm{pp}$.

[11] - On large values of Diriohlet polynomials, to appear in the Procoedings of the Colloquium on Number Theory, Debrecen 1974, North.Holland Publ. Comp.

[12] H. L. Montgomery, Mean and large valabes of Dirichlet polynomials, Inventiones Math. 8 (1969), pp. 334-345.

[13] $-Z$ eros of L-functions, ibid., 8 (1969), pp. 346-354.

[14] - Topics in multiplicative number theory, Lecture Notes in Math. 227, Berlin-Heidelberg-New York 1971.

[15] K. Ramachandra, Some new density estimates for the zeros of the Riemann zeta-function, Ann. Acad. Sci. Fenn. Ser. A. I. Math. 1 (1975), pp. 177-182.

[16] - A simple proof of the mean fourth power estimate for $\zeta\left(\frac{1}{2}+i t\right)$ and $L\left(\frac{1}{2}+i t, \chi\right)$, Ann. Scuola Norm. Sup. Pisa l (1974), pp. 81-97.

DEPARTMENT OF MATHEMATICS

UNIVERSITY OF TUREU

Turku, Finland

\section{The algebraic independence of certain numbers to algebraic powers}

by

W. D. Brownawaid* (Boulder, Col.) and M. Watdosmmidt (Paris)

Dedicated to Professor Th. Schneider on the occasion of his 65 th birthday

In 1949, A. O. Gelfond proved ([4], Theorem 1, pp. 132-133) that if $a$ is an algebraic number $(\alpha \neq 0, \log \alpha \neq 0)$ and $\beta$ is a cubic irrational number, then the two numbers $\alpha^{\beta}$ and $\alpha^{\beta^{2}}$ are algebraically independent (over $\boldsymbol{Q}$ ). Shortly thereafter Gelfond and N. I. Feldman [5] gave a measure of algebraic independence of these two numbers. $R$. Wallisser has conjectured that, for $\beta$ a cubic irrational, $\alpha^{\beta}$ and $a^{\beta^{2}}$ are algebraically independent even when $\alpha$ is only well-approximated by algebraic numbers. In this paper, we establish Wallisser's conjecture when $\alpha$ is closely approximated by algebraic numbers of bounded degree. We wish to thank M. Mignotite for his helpful conments on an earlier draft of this paper.

Trmonem. Let a be a complex number, $\alpha \neq 0, \log \alpha \neq 0$, and $\beta$ a cubio irrational number. Let $f: \boldsymbol{N} \rightarrow \boldsymbol{R}$ with $f \nrightarrow \infty$ and let $d_{0} \in \boldsymbol{N}$. Assume that for infinitely many $T \in \boldsymbol{N}$, there exist algebraic numbers $a_{Y}$ of degree $\leqslant d_{0}$ satisfying

$$
\begin{gathered}
\operatorname{logheight} a_{T} \leqslant T, \\
\log \left|\alpha-a_{T}\right|<-e^{T f(T)} .
\end{gathered}
$$

Then the two numbers $\alpha^{\beta}$ and $\alpha^{\beta^{2}}$ are algebraically independent.

Remark 1. If $\alpha$ itself is algebraic, we let $a=a_{T}$ for $T \geqslant \log$ height $\alpha$.

Remark 2. If $\alpha$ is a complex number $(\alpha \neq 0, \log \alpha \neq 0)$ and $\beta$ a cubic irrational number, with $\alpha^{\beta}, \alpha^{\beta^{2}}$ algebraically dependent, then for all $a_{0} \in \boldsymbol{N}$ there exist two positive constants

$$
O=O\left(\alpha, \beta, a_{0}\right), \quad H=H\left(\alpha ; \beta, d_{0}\right)
$$

* Research partially supported by the National Science Foundation. 
such that, if $a$ is an algebraic number of degree $\leqslant d_{0}$ and of height $\leqslant h$, with $h \geqslant H$, then

$$
\log |\alpha-a|>-h^{c} \text {. }
$$

(In fact, $O$ and $H$ are effectively computable constants.)

EXAMPLE. For $\left.\alpha=\sum_{n=0}^{\infty}(-1)^{n} 2^{-2^{2} .^{.}}\right\}^{2}{ }^{2 n \text { times }}, \alpha^{\beta}$ and $\alpha^{\beta^{2}}$ are algebraically independent for every cubic irrational number $\beta$.

Notations. We fix any cletermination of logarithm in the disk $|z-\alpha|<\alpha$ such that $\log \alpha \neq 0$. When $a$ belongs to this disk, we write $a^{A}$ instead of $\exp (\beta \log a)$.

When $\lambda=\left(\lambda_{0}, \lambda_{1}, \lambda_{2}\right) \in Z^{3}$, we write $\lambda \beta$ instead. of $\lambda_{0}+\lambda_{1} \beta+\lambda_{2} \beta^{2}$ and we define $|\lambda|$ to be $\max \left|\lambda_{i}\right|$. For convenience, any $\lambda, v$ will have nonnegative coordinates unless expressly allowed to be negative (does not apply to $\boldsymbol{\mu}$.

The letters $c_{1}, c_{2}, c_{3}, \ldots$ will denote positive constants which are independent of $T$.

\section{Auxiliary lemmas}

Lemma 1 (Siegel's Lemma). Let $R$ and $S$ be positive integers, $2 R<S$ and. let $a_{i j} \in \boldsymbol{Z}[X], 1 \leqslant i \leqslant R, 1 \leqslant j \leqslant S, A \geqslant 1$, satisfy

$$
\operatorname{deg} a_{i j} \leqslant \delta, \quad \text { height } a_{i j} \leqslant A .
$$

Then there exist polynomials $f_{1}, \ldots, f_{s} \in \boldsymbol{Z}[X]$, not all zero, satisfying

$$
\operatorname{deg} f_{j} \leqslant \delta, \quad \text { height } f_{j} \leqslant\left((1+\delta)^{2} S A\right)^{2 R /\left(S^{\prime}-2 R\right)}
$$

and

$$
\sum_{j=1}^{s} a_{i j} f_{j}=0 \quad \text { for } \quad 1 \leqslant i \leqslant R .
$$

For a proof, see [2], Lemma 5.2.

Lemma 2 (Gelfond). Suppose $P, Q \in C[X]$. Then

$$
\text { (height } P Q) \cdot e^{\text {dog } P Q} \geqslant(\text { height } P \text { ) (height } Q) \text {. }
$$

For a proof (of a more general resulti), sęe [4], Lemma 2, p. 135 or. [6], Lemma 3 , p. 149 .

Lemma 3 (Tijdeman). Suppose $F^{\prime}(z)=\sum_{|v|<N} A_{\nu} e^{(v-p) s}$, and set

$$
\begin{gathered}
b=\max _{\substack{|v|<N \\
b_{0}=}}\{1,|(\boldsymbol{v} \cdot \boldsymbol{\beta})| \cdot \max (1,|\log \alpha|)\}, \\
\min _{|\mu|<N}\{1,|(\boldsymbol{\mu} \cdot \boldsymbol{\beta})| \cdot \min (1,|\log \boldsymbol{\alpha}|)\}, \\
E=\max _{\substack{|\lambda|<L \\
0 \leqslant p<P}}\left|F^{(p)}((\boldsymbol{\lambda} \cdot \boldsymbol{\beta}) \log \boldsymbol{\alpha})\right| .
\end{gathered}
$$

Then, if $L \leqslant N$ and $P L^{3} \geqslant 2 N^{3}+13 b^{2}$, we have

$$
\max \left|A_{\nu}\right| \leqslant L^{3} \sqrt{\left(N^{3}\right) !} e^{7 b^{2}}\left(\frac{1}{2 b_{0} b}\right)^{N^{3}}\left(\frac{72 b}{b_{0} L^{3 / 2}}\right)^{P L^{3}} B .
$$

([7], Theorem 3, pp. 87-88.)

LFimma 4. Let $f(X), g(X) \in \mathbb{Z}[X]$ have heights $|f|$ and $|g|$, respectively, and degrees $m$ and $n$, respectively. Then $f(X)$ and $g(X)$ have a common divisor in $Z[X]$ if and only if for some $\omega \in \boldsymbol{C}$,

$$
\max \{|f(\omega)|,|\dot{g}(\omega)|\} \leqslant|f|^{-n}|g|^{-m}(m+n)^{-(m+n)} .
$$

For a proof, see [4:], Lemma V, pp. 14:5-146 or [1], Lemma 1, p. 14.

Lemrara 5. Suppose $\omega \in \boldsymbol{C}$ and $P(X) \in \boldsymbol{Z}[X]$ satisfy $|P(\omega)|<e^{-\lambda \dot{a}(h+d)}$, where $\lambda \geqslant 3, d=\operatorname{deg} P$, height $P=e^{h}$. Then there is a factor $Q(X)$ of $P(X)$ which is a power of an irreducible polynomial in $\mathbf{Z}[X]$ such that

$$
\log |Q(\omega)|<-(\lambda-1) d(h+d) .
$$

For a proof, see [4], Lemma VI, p. 147 or [1], Lemma 3 , pp. $15-16$ Limoxa 6. Suppose $\omega \in C$ is transcendental, $\xi \in C$ is algebraic integral over $\boldsymbol{Z}[\omega]$, of degree $\delta$, having a minimal polynomial (over $\boldsymbol{Z}[\omega]$ ) with coefficients of degree $\leqslant d$ and height $\leqslant e^{h}$. Let $\lambda_{1}, \lambda_{2}$ be two real numbers satisfying

If

$$
\lambda_{1}>\lambda_{2}>6+2 \log (\delta+1)+2 \log (|\omega|+1) .
$$

$$
-\lambda_{1} d(h+d) \leqslant \log |\xi| \leqslant-\lambda_{2} d(h+d)
$$

then there exist an irreducible polynomial $P(\omega) \in \boldsymbol{Z}[\omega]$, and an integer $s \geqslant 1$, such that $P^{s}$ divides the norm of $\xi$ over $Q(\omega)$, and that

$$
-3 \delta \lambda_{1} d(h+d) \leqslant \log |P(\omega)| \leqslant-\frac{\lambda_{2}}{6 s} d(h+d) \text {. }
$$

Proof of Lemma 6. The proof is an adaptation of Ohudnovskii's arguments in [3].

Consider the minimal polynomial of $\xi$ over $\boldsymbol{Z}[\omega]$ :

$$
\xi^{\delta}+u_{\delta-1}(\omega) \xi^{\delta-1}+\ldots+u_{0}(\omega)=0,
$$

with $u_{i}(\omega) \epsilon \boldsymbol{Z}[\omega]$, deg $u_{i} \leqslant d$, height $u_{i} \leqslant e^{h}(0 \leqslant i \leqslant 8-1)$.

The norm of $\xi$ over $Q(\omega)$ is $u_{0}(\omega)$, and

$$
\begin{gathered}
u_{0}(\omega)=-\xi \sum_{i=1}^{\delta} u_{i}(\omega) \xi^{i-1} \quad\left(\text { with } u_{\delta}=1\right), \\
\left|u_{0}(\omega)\right| \leqslant|\xi| \delta(d+1) e^{h} \max \left\{1,|\omega|^{d}\right\},
\end{gathered}
$$


since $|\xi|<1$. Consequently

$$
\begin{aligned}
\log \left|u_{0}(\omega)\right| & \leqslant-\lambda_{2} d(h+d)+h+d \log \max \{1,|\omega|\}+\log \delta(d+1) \\
& <-\frac{1}{2} \lambda_{2} d(h+d) .
\end{aligned}
$$

By Lemma 5 , we get an irreducible polynomial $P(\omega) \in Z[\omega]$, and an integer $s \geqslant 1$, such that $P^{s}(\omega)$ divides $u_{0}$, and

$$
\log \left|P^{s}(\omega)\right|<-\left(\frac{\lambda_{2}}{2}-1\right) d(h+d)<-\frac{\lambda_{2}}{6} d(h+d) .
$$

We show that

$$
\log |P(\omega)|>-3 \delta \lambda_{1} \partial(h+d)
$$

by deriving a contradiction from the contrary assumption.

Assume

$$
\log |P(\omega)| \leqslant-3 \delta \lambda_{1} d(h+d) .
$$

We claim that for each $j(0 \leqslant j \leqslant \delta-1)$,

$$
\log \left|u_{j}(\omega)\right| \leqslant-2 \delta \lambda_{1} d(h+d) .
$$

The claim is true for $j=0: P(\omega)$ divides $u_{0}(\omega)$, and the quotient has degree $\leqslant d$ and height $\leqslant e^{h+d}$ (using Liemma 2 ), hence has absolute value $\leqslant(d+1) e^{h+d} \max \left\{1,|\omega|^{d}\right\}$.

We now assume that the claim is true up to $j-1$, with $1 \leqslant j \leqslant \delta-1$, and, under that assumption, prove it true for $j$. We can write

$$
-u_{j}(\omega)=\xi^{\delta-j}+\sum_{i=0}^{j-1} \frac{u_{i}(\omega)}{\xi^{j-i}}+\xi \sum_{l=j+1}^{\delta-1} \xi^{l-j-1} u_{l}(\omega) .
$$

Vsing the induction hypothesis, and the lower bound for $|\xi|$, we deduce

$$
\log \frac{\left|a_{i}(\omega)\right|}{|\xi|^{j-i}} \leqslant-(2 \delta-j+i) \lambda_{1} d(h+d) \leqslant-\delta \lambda_{1} d(h+d)
$$

for $0 \leqslant i \leqslant j-1$. Since

$$
\left|u_{l}(\omega)\right| \leqslant(d+1) e^{h} \max \left\{1,|\omega|^{[\pi}\right\}, \quad j+1 \leqslant l \leqslant \delta-1,
$$

we obtain

$$
\log \left|u_{j}(\omega)\right| \leqslant-\frac{1}{2} \lambda_{2} d(h+d)
$$

Consequently, by Lemma $4, u_{j}(\omega)$ and $P^{s}(\omega)$ have a common factor, i.e. $P(\omega)$ divides $u_{f}(\omega)$ in $\boldsymbol{Z}[\omega]$. As a result:

$$
\log \left|u_{j}(\omega)\right| \leqslant-2 \delta \lambda_{1} d(h+d)
$$

and the claim follows.
However, the claim gives

$$
\left.|\xi|^{\delta} \leqslant \sum_{j=0}^{\delta-1} \mid u u_{j}(\omega)\right)\left.|| \xi\right|^{j-1} \leqslant \delta e^{-2 \delta \lambda_{1} d(h+a)}
$$

and consequentily

$$
\log |\xi| \leqslant 1-2 \lambda_{1} d(h+d)<-\lambda_{1} d(h+d),
$$

contradicting the hypothesis.

This contradiction completes the proof of Lemma 6 .

Proof of the theorem. The proof is based on the ideas of Gelfond and N. I. Feldman' [5], and G. V. Ohudnovskii [3]. It follows from Gelfond's transcendence measure ([4], Theorem III, p. 134)-for numbers of the type $a^{b}$, where $a$ and $b$ are algebraic numbers with $\log a \neq 0, b$ irrational, that both numbers $a^{\beta}$ and $\alpha^{\beta^{2}}$ are transcendental. Suppose that the field $\boldsymbol{Q}\left(a^{\beta}, \alpha^{\beta^{2}}\right)$ has'transcendence degree one. Then we can write $\boldsymbol{Q}\left(\beta, \alpha^{\beta}, \alpha^{\beta^{2}}\right)$ $=Q\left(\omega, \omega_{1}\right)$, where $\omega$ is transcendental (we can choose $\left.\omega=\alpha^{\beta}\right)$, and $\omega_{x}$ is integral over $\boldsymbol{Z}[\omega]$ of degree $m$. Let $v \in \boldsymbol{Z}[\omega], v \neq 0$, such that $v \alpha^{\beta}, v \beta^{\beta^{1}}$, $v \alpha^{-\beta}$ and $v \alpha^{-\beta^{2}}{ }_{\epsilon} \boldsymbol{Z}\left[\omega, \omega_{1}\right]$. We may assume without loss of generality that $\beta$ is an algebraic integer and that $f(T) \leqslant \log T$.

Let $T$ be a sufficiently large positive integer with $a_{T}$ an algebraic number satisfying the hypotheses of the theorem. Then select $\Delta \in \boldsymbol{N}$, $1 \leqslant \Delta \leqslant e^{T}$, such that $\Delta a_{T}$ is an algebraic integer and set

$$
N_{0}=[\exp T f(T) / 7], \quad N_{1}=\left[N_{0}^{2} \log N_{0}\right]
$$

It is easy to check that

$$
N_{1}^{3} \log N_{1} \leqslant e^{13 T f(T) / 14}
$$

For $N \in \boldsymbol{N}$ with $N_{0} \leqslant N \leqslant N_{1}$, we define

$$
\begin{aligned}
& t_{N}=\left[N^{1 / 2} f(T)^{1 / 4}\right], \\
& H_{N}=\left[N^{3 / 2}(\log N) f(T)^{-9 / 4}\right], \\
& P_{N}=\left[c_{1} D^{3 / 2} f(T)^{-3 / 4}\right],
\end{aligned}
$$

where $c_{1}=1 /\left(4 d_{0} m\right)$

The inequality $N L_{N} T \leqslant 8 H_{N}$. which follows from our assumption that $T f(T) \leqslant 7 \log N$, will be used repeatedly below, often without mention.

STMP 1. We show that there exist elements $\varphi(\boldsymbol{v}) \in \boldsymbol{Z}[\omega],|\boldsymbol{v}|<N$, not all of which are zero and without a common divisor in $\mathbf{Z}[\omega]$, satisfying

$$
\operatorname{logheight} \varphi(\boldsymbol{v}) \leqslant 0_{2} H_{N}, \quad \operatorname{deg} \varphi(\boldsymbol{v}) \leqslant c_{3} L_{N} \mathcal{N},
$$

such that the function

$$
F_{N}^{\prime}(z)=\sum_{|v|<N} l \varphi(\boldsymbol{v}) \exp ((\boldsymbol{v} \cdot \boldsymbol{\beta}) z)
$$


satisfies

$$
\log \left|F_{N}(z)\right|_{|z|=N^{3 / 2}} \leqslant-c_{4} N^{3} \log N
$$

Proof. (i) Consider the numbers

$$
\Phi_{p, \lambda}=\sum_{|\boldsymbol{v}|<N} \varphi(\boldsymbol{v})(\boldsymbol{v} \cdot \boldsymbol{\beta})^{p p} a_{T^{\mu}}^{\mu_{0}} a^{\beta \mu_{1}} \alpha^{\beta^{2} \mu_{2}} \quad\left(0 \leqslant p<P_{N}\right)
$$

(iii) We now use Hermite's interpolation formula on the circles about the origin of radii $N^{3 / 2}$ and $N^{2}$. For $N^{3 / 2} \leqslant|z|<N^{2}$, we have

$$
\begin{aligned}
F_{N}(z)= & \frac{1}{2 \pi i} \int_{|\zeta|=N^{2}} \frac{F_{N}(\zeta)}{\zeta-z} \prod_{\lambda}\left(\frac{z-(\lambda \cdot \beta) \log \alpha}{\zeta-(\lambda \cdot \beta) \log \alpha}\right)^{P_{N}} d \zeta- \\
& -\frac{1}{2 \pi i} \sum_{\lambda} \sum_{p=0}^{P_{N}-1} \frac{Z_{N}^{(p)}((\lambda \cdot \beta) \log \alpha)}{p !} \times \\
& \times \int_{|\zeta-\lambda \cdot \beta \log \alpha|=m b / 2} \frac{(\zeta-\lambda \cdot \beta \log \alpha)^{p}}{\zeta-z} \prod_{\lambda^{\prime}}\left(\frac{z-\lambda^{\prime} \cdot \beta \log \alpha}{\zeta-\lambda^{\prime} \cdot \beta \log \alpha}\right)^{P_{N}} d \zeta
\end{aligned}
$$

where $\mu_{0}, \mu_{1}, \mu_{2} \in \boldsymbol{Z}$ and $\mu_{0}+\mu_{1} \beta+\mu_{2} \beta^{2}=\langle\boldsymbol{v} \cdot \boldsymbol{\beta}\rangle(\boldsymbol{\lambda} \cdot \boldsymbol{\beta})$, for $|\lambda|<I_{N}$, and the $\varphi(v)$ satisfy

$$
\operatorname{logheight} \varphi(v) \leqslant c_{2} H_{N}, \quad \operatorname{deg} \varphi(v) \leqslant c_{3} L_{N} N .
$$

The numbers

$$
\left(v^{c_{5}} \Delta\right)^{N L_{N} \Phi_{p, \lambda}}
$$

are polynomials in $\omega_{1}$ and $\Delta a_{T}$ with coeffieients from $\boldsymbol{Z}[\omega]$. These coefficients themselves have degree $\leqslant c_{6} N L_{N}$ and

$$
\text { logheight } \leqslant c_{7}\left(N L_{N} \operatorname{logheight} a_{T}+H_{N}\right) \leqslant c_{8} H_{N}
$$

by our choice of range of $N$. We want to choose the $p(\boldsymbol{y})$ such that the coefficients of the at most $d_{0} m$ monomials in $\omega_{1}$ and $\Delta a_{T}$ vanish for $0 \leqslant p<P_{N}$ and $|\lambda|<L_{N}$. The number of equations is at most

$$
d_{0} m P_{N} L_{N}^{3} \leqslant N^{3} / 4
$$

and the number of unknowns is $N^{3}$. Thus by Lemma 1 the system has a non-trivial solution with $p_{0}(\boldsymbol{v}) \in \boldsymbol{Z}[\omega]$ satisfying

$$
\operatorname{deg} \varphi_{0}(v) \leqslant c_{3} N L_{N}, \quad \operatorname{logheight} \varphi_{0}(v) \leqslant c_{9} H_{N}
$$

After dividing each $\varphi_{0}(v)$ by the greatest common divisor of all the $\varphi_{0}(v)$, Lemma 2 assures us that the quotients $p(v)$ satisfy

$$
\operatorname{deg} \varphi(\boldsymbol{y}) \leqslant c_{3} N L_{N}, \quad \operatorname{logheight} \varphi\langle\boldsymbol{v}\rangle \leqslant c_{9} H_{N}+c_{3} N L_{N} \leqslant c_{2} H_{N}
$$

as desired.

(ii) For $0 \leqslant p<P_{N}$ and $|\lambda|<L_{N}$, we have

$$
\left|T_{N}^{(p)}((\lambda \cdot \boldsymbol{\beta}) \log \alpha)-\Phi_{p, \lambda}\right| \leqslant \sum_{\nu}|\varphi(v)||\boldsymbol{v} \cdot \boldsymbol{\beta}|^{p}\left|\alpha^{\beta}\right|^{\mu_{1}}\left|\alpha^{\beta^{2}}\right|^{\mu_{2}}\left|\alpha^{\mu_{0}}-a_{g^{0}}^{\mu_{0}}\right|
$$

So

But since

$$
\log \left|F_{N}^{(2)}((\lambda \cdot \beta) \log \alpha)\right| \leqslant e_{10}\left(H_{N}+p \log N+N L_{N} T\right)+\log \left|\alpha-a_{q^{T}}\right|
$$

$H_{N}+p \log N+N L_{N} T \leqslant 10 H_{N} \leqslant 10 H_{N_{1}}<N_{1}^{3} \log N_{1} \leqslant \exp (13 T f(T) / 14)$ we have

$$
\log \left|F_{N}^{(p)}((\lambda \cdot \boldsymbol{\beta}) \log \alpha)\right| \leqslant-\frac{1}{2} \exp (T f(T)) \leqslant-\left(\sqrt{N_{0}} / 2\right) N^{3} \log N
$$

un over all $\lambda, \lambda^{\prime}$ with coordinates between 0 and $L_{N-1}$ where the indices run over all $\lambda, \lambda^{\prime}$ with coordinates between 0 and $L_{N}-1$,
and where $b=|\log \alpha| \min _{\lambda \neq \lambda^{\prime}}\left|\lambda \cdot \boldsymbol{\beta}-\lambda^{\prime} \cdot \boldsymbol{\beta}\right|$. To estimate

$$
\left|F_{N}\right|_{N^{3 / 2}}=\max _{|z|=N^{3 / 2}}\left|F_{N}(z)\right|
$$

we use the following bounds:

$$
\log \left|F_{N}\right|_{N^{2}} \leqslant c_{11}\left(H_{N}+N^{3}\right)<c_{12} N^{3},
$$

$$
\begin{aligned}
& \underset{\substack{|z|=N^{3 / 2} \\
|\zeta|=N^{2}}}{\log \operatorname{sim}_{2}} \prod_{\mid}\left|\frac{z-\lambda \cdot \beta \log \alpha}{\zeta-\lambda \cdot \beta \log \alpha}\right|^{P_{N}} \leqslant-L_{N}^{3} P_{N} \log \frac{N^{2}}{3 N^{3 / 2}} \leqslant-\frac{c_{1}}{6} N^{3} \log N, \\
& \log b \geqslant-c_{13} \log N,
\end{aligned}
$$

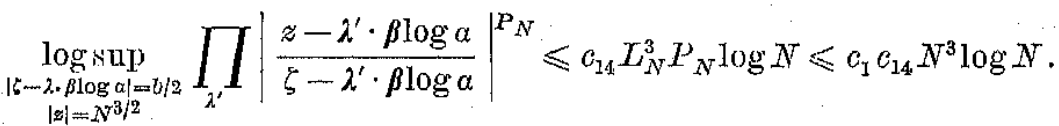

Thus we obtain

$$
\log \left|F_{N}\right|_{N^{3 / 2}} \leqslant-c_{4} N^{3} \log N
$$

StEP 2. We now note that there exist $p_{0} \in \boldsymbol{Z}, P_{N} \leqslant p_{0} \leqslant\left[\frac{3}{c_{1}}\right] P_{N}-1$ and $\lambda \in \boldsymbol{Z}^{3}$ with $|\lambda|<L_{N}$, such that

$$
-c_{15} N^{3} \log N \leqslant \log \left|F_{N}^{\left(p_{0}\right)}(\lambda \cdot \beta \log \alpha)\right| \leqslant-c_{16} N^{3} \log N .
$$

The upper bound

$$
\log \left|F_{N}^{\left(p_{0}\right)}(\lambda \cdot \beta \log \alpha)\right| \leqslant-c_{16} N^{3} \log N
$$

is a direct consequence of Step 1 and Cauchy's integral formula. Assume that, for every pair $p_{0}, \lambda$ in the considered ranges,

$$
\log \left|F_{N}^{\left(2_{0}\right)}(\lambda \cdot \beta \log \alpha)\right| \leqslant-c_{15} N^{3} \log N
$$


for some large $c_{15}$. Then, by Lemma 3 ,

$$
\log \max |\varphi(\boldsymbol{v})| \leqslant-c_{17} N^{3} \log N, \quad \text { with } c_{17}>0 .
$$

For each $\boldsymbol{v}$ with $\phi(\boldsymbol{v}) \neq 0$, we choose by Lemma 5 a factor $q(\boldsymbol{v})$ of $\varphi(\boldsymbol{y})$ in $\boldsymbol{Z}[\omega]$ such that $q(\boldsymbol{v})$ is a power of an irreducible polynomial in $\boldsymbol{Z}[\omega]$ and

$$
\begin{gathered}
\log |q(\boldsymbol{v})| \leqslant-c_{18} N^{3} \log N, \\
\operatorname{deg} q(\boldsymbol{v}) \leqslant c_{3} N L_{N}, \quad \operatorname{logh\operatorname {height}} q(\boldsymbol{v}) \leqslant c_{19} H_{N} .
\end{gathered}
$$

Since the $\varphi(v)$ do not all have a common factor in $\mathbf{Z}[\omega]$, at least two of the $q(v)$ must be powers of distinct irreducible polynomials, contradieting Lemmar 4 , since

$$
2 c_{3} c_{19} N L_{N} H_{N}+2 c_{3} N L_{N} \log \left(2 c_{3} N L_{N}\right) \leqslant c_{20} N^{3}(\log N) f(T)^{-1 / 2} .
$$

We now know that $\Phi_{p_{0}, 2}$ and hence $\left(v^{c_{5}} \Delta\right)^{c_{21} N L_{N}} \Phi_{p_{0}, 2}$ satisfy

$$
-c_{22} N^{3} \log N \leqslant \log |w| \leqslant-c_{23} N^{3} \log N \text {, }
$$

by the argument of Step 1 (ii).

STIE 3. The number $\xi_{N}=\left(v^{c_{5}} \Delta\right)^{c_{21} N L_{N}} \Phi_{p_{0}, \lambda}$ and its conjugates over $\boldsymbol{Q}(\omega)$ are polynomials in the conjugates of $\omega_{1}$ and $\Delta a_{T^{\prime}}$ over $\boldsymbol{Q}(\omega)$ (of degrees $\leqslant m$ and $d_{0}$ respectively), with coefficients in $\boldsymbol{Z}[\omega]$ having

$$
\text { degree } \leqslant c_{24} N L_{N}, \quad \operatorname{logheight} \leqslant c_{25} H_{N} .
$$

Using Lemma 6, we get an irreducible potynomial $R_{N}(\omega)$ in $\boldsymbol{Z}[\omega]$ and an integer $s_{N} \geqslant 1$, such that $R_{N}(\omega)$ and $Q_{N}(\omega)=R_{N}(\omega)^{B_{N}}$ satisfy

$$
\operatorname{deg} Q_{N} \leqslant c_{24} N L_{N} ; \quad \operatorname{logheight} Q_{N} \leqslant c_{26} H_{N} ;
$$$$
-N^{3}(\log N) f(T)^{1 / 4}<\log \left|R_{N}(\omega)\right| ; \quad \log \left|Q_{N}(\omega)\right| \leqslant-c_{27} N^{3} \log N .
$$

STEP 4. We apply Lemma 4 to the polynomials $Q_{N}(\omega)$ and $Q_{N+1}(\omega)$, for $N_{0} \leqslant N<N_{1}$. We estimate

$$
\begin{gathered}
\operatorname{deg} Q_{N} \cdot \operatorname{logheight} Q_{N+1}+\operatorname{deg} Q_{N+1} \cdot \operatorname{logheight} Q_{N}+ \\
+\left(\operatorname{deg} Q_{N}+\operatorname{deg} Q_{N+1}\right) \cdot \log \left(\operatorname{deg} Q_{N}+\operatorname{deg} Q_{N+1}\right) \\
\leqslant c_{28} N L_{N} H_{N} \leqslant c_{28} N^{3}(\log N) f(T)^{-1 / 2}
\end{gathered}
$$

Oonsequently, by Lemma $4, Q_{N}(\omega)$ and $Q_{N+1}(\omega)$ have a common factor: $R_{N}(\omega)=R_{N+1}(\omega)$, since both polynomials are powers of irreducible polynomials in $\boldsymbol{Z}[\omega]$.

Consequently

$$
R_{N_{1}}=R_{N_{0}} \quad \text { and } \quad Q_{N_{1}}(\omega)=R_{N_{0}}^{s_{N_{1}}}(\omega)
$$

thus

$$
\begin{aligned}
-\log \left|Q_{N_{1}}(\omega)\right| & =-s_{N_{1}} \log \left|R_{N_{0}}(\omega)\right|<c_{24} N_{1}^{3 / 2} f(T)^{1 / 4} N_{0}^{3}\left(\log N_{0}\right) f(T)^{1 / 4} \\
& \leqslant c_{29} N_{1}^{3 / 2} f(T)^{1 / 4} N_{1}^{3 / 2}\left(\log N_{1}\right)^{-3 / 2}\left(\log N_{1}\right) f(T)^{1 / 4} \\
& \leqslant c_{29} N_{1}^{3}\left(\log N_{1}\right)^{-1 / 2} f(T)^{1 / 2}
\end{aligned}
$$

which contradicts the upper bound on $\left|Q_{N_{1}}(\omega)\right|$ found in Step 3.

The contradiction establishes the theorem.

\section{References}

[1] W. D. Brown a well, Sequences of Diophantine approximations, J. Number Theory 6 (1974); pp. 11-21.

[2] - Get'fond's method for atgebraio independence, Trans. A. M. S. 205 (1975), pp. 1-26.

[3] G. V. Chudnovikii, Algebraic independence of some values of the exponential funotion (in Russian), Mat. Zametki 5 (1974), pp. 661-672 [English transla. tion: Math. Notes 15 (1974), pp. 391-398).]

[4] A. O. Gelfond, Transcendental and algebraic numbers, Dover, New York 1960.

[5] A. 0 . Gelfond and X. I. Feldman, On the measure of relative transcendence of eertain numbers. (in Russian), Izv. Akad. Nauk S.S.S.R., Ser. mat., 14 (1950), pp. $493-500$.

[6] R. Tijdeman, On the algebraic independenoe of certain numbers, Nederl. Akad. Wet. Proe., Ser. A $74(1971)=$ Indag. Math. 33 (1971), pp. 146-162.

[7] - An auxiliary result in the theory of transcendental numbers, J. Number Theory 5 (1973), pp. 80-94.

\section{DRPARTMENT OF MATHEMATIOS}

UNIVTRSITY OF COLORADO

and

THE PANNSYLVANIA STATE UNIVRRSTTY

Boulder, Colorado, U.S.A.

UNIVERSITH PARIS VI

MATHEMATIQUES 